International Mathematical Forum, 2, 2007, no. 1, 1 - 20

\title{
On concepts of PDE-Software: The cellwise oriented approach in DEAL.
}

\author{
F.T. Suttmeier \\ Fachbereich Mathematik \\ Universität Siegen \\ D-57258 Siegen \\ Germany
}

\begin{abstract}
In this note, we review and refine our concepts of a cellwise oriented approach to PDE-software described in the dissertations of G. Kanschat and F.T. Suttmeier. The problem of non-matching cells in finite element discretisations on locally refined meshes is adressed. General concepts of the treatment of so-called hanging nodes are illustrated at the standard bilinear and -quadratic FE-spaces. These results carry over to nonconforming elements.

Furthermore, two features we focus on are on one hand the significant reduction of memory requirements employing local scaling techniques for representing operators. The second aspect is data locality, which is the basis for vectorisation/parallelisation and for employing efficient cache-optimised hardware-techniques.

We illustrate our ideas and provide numerical results by treating a standard benchmark problem (disc with a hole) from structural mechanics.
\end{abstract}

Mathematics Subject Classification: 65N50, 65N99

Keywords: adaptive finite elements, PDE-software

\section{Introduction}

DEAL (Differential Equations Analysis Library) is available via [2]. This $\mathrm{C}++$ class library for finite elements was designed by Guido Kanschat and FranzTheo Suttmeier in 1992. The implementation was continuously improved between 1993 and 1998 by G. Kanschat, F.T.Suttmeier and R. Becker.

With DEAL as the heart in research on adaptive FEM at the University of Heidelberg, pioneering work on the so called Dual Weighted Residual (DWR) 
approach was done by R. Rannacher and his co-workers in R. Becker [1], G. Kanschat [4] and F.T.Suttmeier [8].

The following text is partly taken from [4]: Scientific computing software to solve "real life" problems tends to become more and more complex. The necessary validation of program code becomes a hard task by this development. A proof of correctness is possible only for simple data structures and small programs. The classic approach to more reliable software used in numerical computations is modular programming. Applying this paradigm, a complex algorithm is decomposed into small simple parts which can be tested independently. But, as mentioned above, not only methods are complex, but data structures too. Object oriented programming now allows the modularization of algorithms and data by combining both of these aspects into the same structure. This leads to the notion of classes and objects.

The tight coupling of data and methods operating on them in object oriented programming, forces design to become an important step in software development. In previous numerical codes, this has been regarded only with the aim of reducing memory usage and computation time.

In a complex piece of software, design of classes has to optimize a combination of four development aims:

1. computing speed,

2. memory requirements,

3. verifiability of code and

4. flexibility.

While the first two points have been investigated very thoroughly in the last 30 years - see e. g. BLAS routines - points three and four are real white patches on the map. Additionally to the known trade-off between speed and memory consumption points three and four introduce a much more complicated balance. From the economic point of view we have to supplement run-time efficiency by development efficiency. In particular, software designed to develop new algorithms must obey this point, since implementation time usually exceeds run-time by orders of magnitude.

The main idea to improve flexibility and correctness of a program lies in the restriction of data access. In usual FORTRAN or $\mathrm{C}$ code a great deal of data is handled by common blocks and global variables, respectively. These allow unrestricted read and write access to their members, so the programmer can not be sure where these structures are changed or corrupted. This verification problem is augmented by the fixed structure of implementation: a change in the data structure necessitates a change in all functions using it. 
Considering application to partial differential equations, there are several parts of the algorithms which may be separated to a high degree. There is a base level of classes describing the adaptive grid generation and handling of multi-grid structures. This part of software is invariant for a huge class of finite element problems. A second level on top of the first defines basic numerical operations like application of an operator to a discrete function. Here, the dependence on the structure of the physical problem is very strong. A third part of code provides standard numerical solvers as cg and Bi-cgstab for linear systems and Newton's method for nonlinear problems. This level should be implemented in an abstract way to allow usage for different applications.

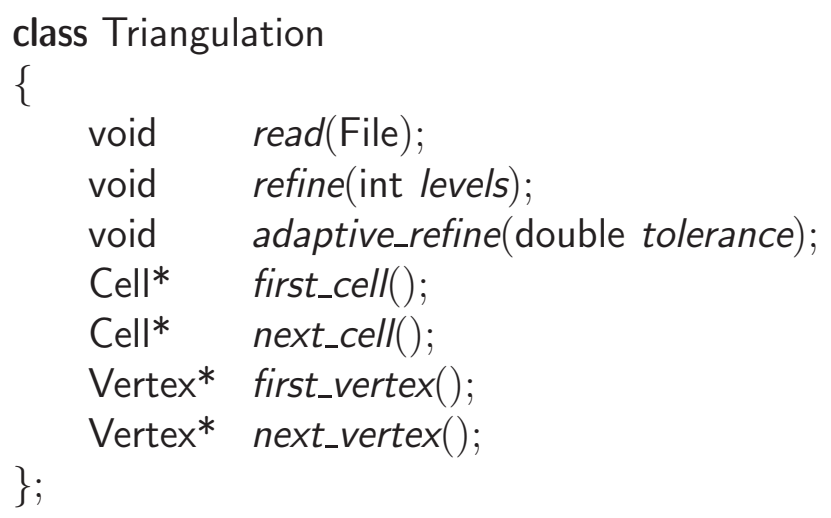

Figure 1: The Triangulation class

\section{Grid handling}

The following text is partly taken from [4]: Let us first regard the abstract concept of a triangulation of a domain $\Omega$ as a hierarchy of grid cells with a certain topology. Its basic functionality is shown in Figure 1, where the triangulation is reduced to a set of cells and vertices with some additional mesh generation functions. This mesh should be able to consist of triangles and quadrangles as well as tetrahedra, prisms and hexahedra in two and three dimensions respectively. This means that we have to extract basic information from all these geometric objects to identify them as a cell. The cell information necessary for adaptive refinement was first collected in [7] in a very abstract way. This led to the definition of a cell essentially following Figure 2.

The function refine of Triangulation works by traversing all cells and forcing each cell to refine itself. Since a correct triangulation is characterized by consistent values of vertices, neighbors and father/child information, refine and coarse function of Cell ensure conservation of these data. Certainly, a cell can hardly supply any of these functions, since topology information is 
specified only for more concrete objects. Therefore, they are declared as an abstract interface and are implemented in derived classes for e. g. triangles and quadrilateral cells, where we know the number of neighbors is three or four respectively.

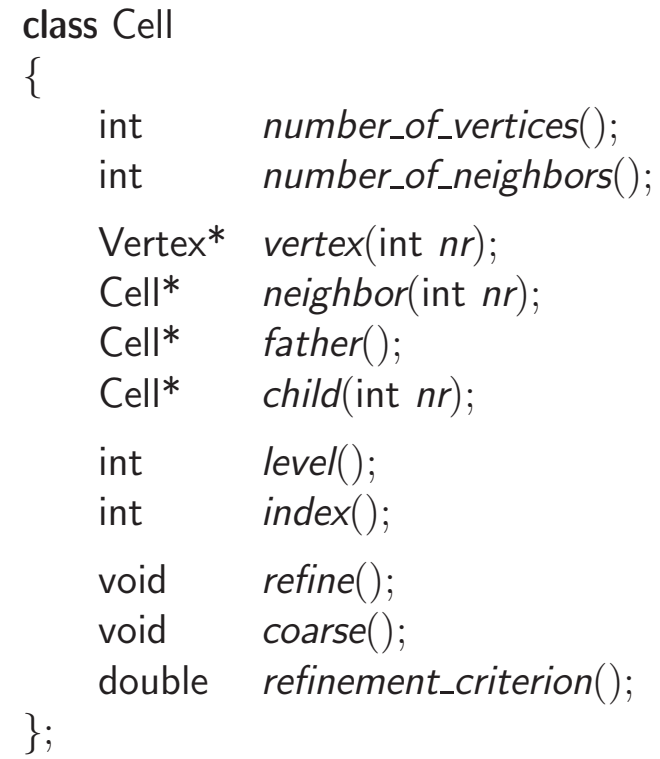

Figure 2: The Cell class (topology and refinement)

The functional interface to these values enables high flexibility regarding implementation. Considering e. g. the children there are two possibilities of obtaining the corresponding pointers. They may be stored in a cell producing memory consumption, but making fast access possible. Alternatively, they can be reconstructed from the list of cells stored in the triangulation. According to the algorithms used as well as preference for memory or speed optimization, these techniques should be chosen appropriately. The interface of Figure 2 allows the necessary internal change of representation on a purely local basis, since implementation details are hidden for other functions. The correctness of working code using this function child is not influenced by internal changes, if only the result of child is correct.

We ensure for each function, that all objects involved in its operation not only explicitly modified objects - are in an admissible state after the function returns control to the calling context.

Take e. g. cell refinement as shown in Figure 3. We start with a valid locally refined triangulation (a). The cells are linked by the mutual neighborship relation $\circlearrowleft$ and the "is child of" relation $\downarrow$. We display two consecutive layers of refinement on top of each other. After splitting the middle cell $(*)$ into four children, the topology is corrupted (b). Setting topology information 
for the children (c), the cell we operated on is valid, but the operation has destroyed the triangulation structure: there is only an unidirectional neighborship relation $\curvearrowright$ from the children of $(*)$ to their neighbors. Finally, the topology information of neighboring cells is updated too and the topology is valid again $(\mathrm{d})$. Compared to other strategies, this process not only avoids expensive postprocessing, but enables us to stop refinement after processing an arbitrary cell (which is actually done in adaptive algorithms). While the states (b) and (c) are "virtual" grids never to be seen in an application, (a) and (d) represent states, where further operations may be inserted.

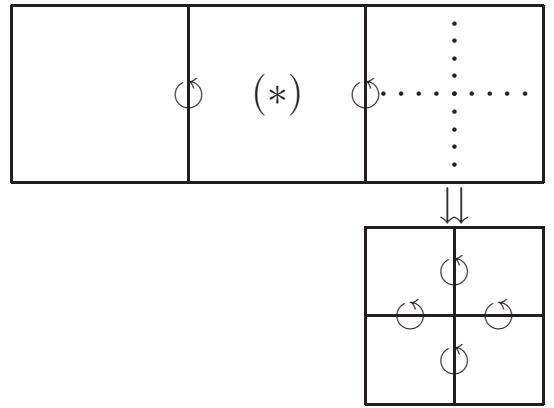

(a) Starting grid with 1 refined cell

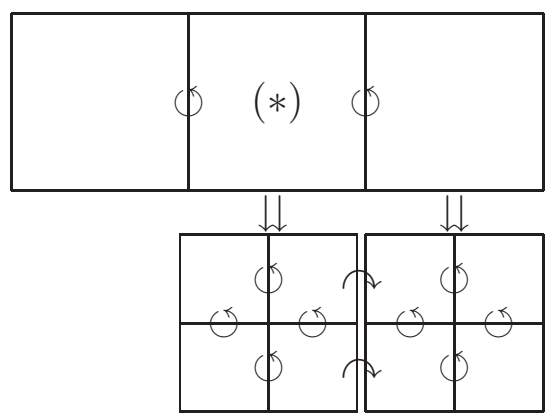

(c) Topology of children

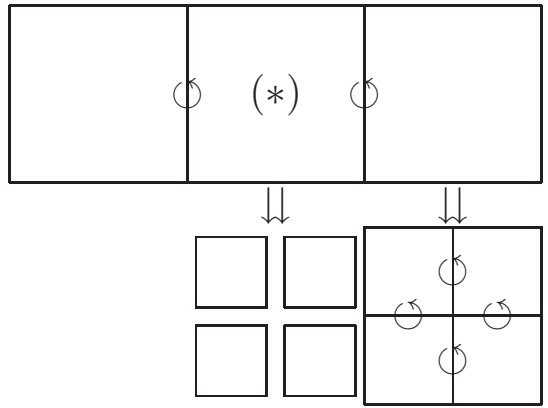

(b) Children generated

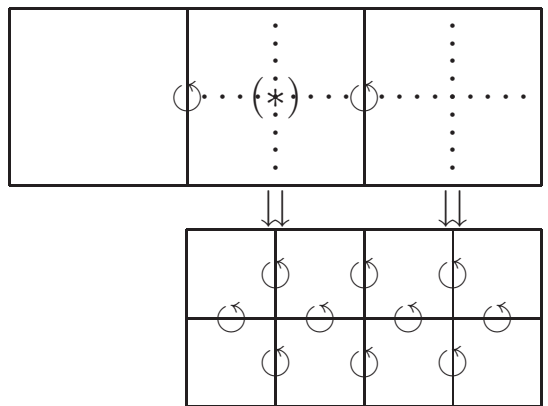

(d) Refined grid

Figure 3: Refinement of a cell

\section{Hanging nodes}

The problem of non-matching cells in finite element discretisations on locally refined meshes is adressed. General concepts of the treatment of so-called hanging nodes are illustrated at the standard bilinear and -quadratic FE-spaces. Furthermore these results carry over to nonconforming elements. 
The Laplace problem

$$
-\Delta u=f \quad \text { in } \Omega, \quad u=0 \quad \text { on } \partial \Omega,
$$

on the domain $\Omega \subset \mathbb{R}^{2}$, is taken as a model problem to present our ideas.

The variational formulation

$$
(\nabla u, \nabla \varphi)=(f, \varphi) \quad \forall \varphi \in V:=H_{0}^{1}(\Omega),
$$

serves as a basis for applying FE methods to (1). Here and below, (., .) denotes the inner product of $L^{2}(\Omega)$ and $\|\cdot\|$ the corresponding norm, while $(\nabla \cdot \nabla \cdot)$ is the natural energy inner product of problem (2). Further, $H^{m}(\Omega)$, for $m \in \mathbf{N}$, denotes the usual $\mathrm{m}$-th order Sobolev space with norm $\|\cdot\|_{m}$, and $H_{0}^{1}(\Omega)$ the subset of $H^{1}(\Omega)$ of functions vanishing on $\partial \Omega$. These are all spaces of either scalar or vector-valued functions and no distinction will be made in the notation of the corresponding inner products and norms. Now, we apply the finite element method on decompositions $\mathbb{T}_{h}=\{T\}$ of $\Omega$ consisting of quadrilaterals $T$, satisfying the usual condition of shape regularity. The width of the mesh $\mathbb{T}_{h}$ is characterised in terms of a piecewise constant mesh size function $h=h(x)>0$, where $h_{T}:=h_{\mid T}=\operatorname{diam}(T)$. Using the notation $V_{h} \approx V$ for the corresponding finite element spaces approximating $V$, the discrete solution $u_{h} \in V_{h}$ is determined by the equation

$$
\left(\nabla u_{h}, \nabla \varphi\right)=(f, \varphi) \quad \forall \varphi \in V_{h} .
$$

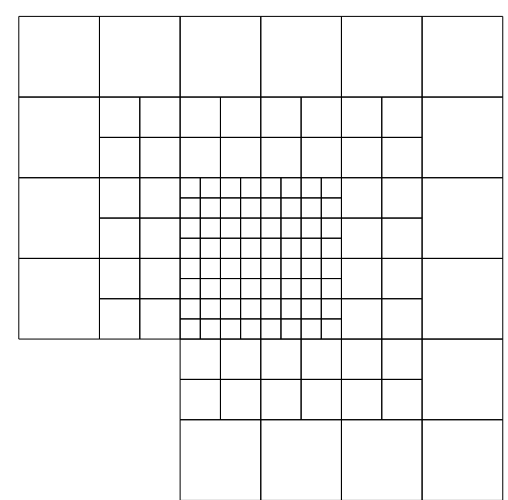

Figure 4: Domain and sample mesh with hanging nodes

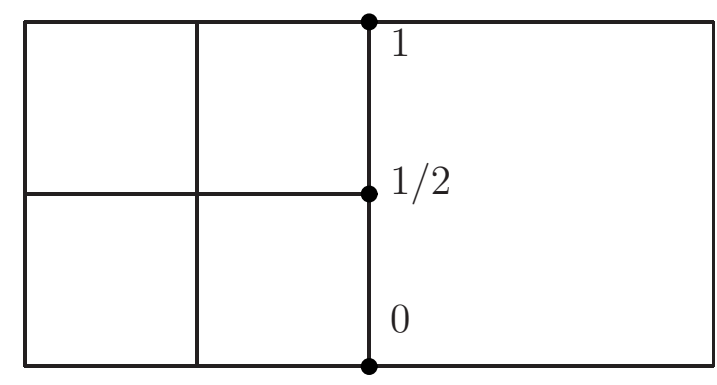

Figure 5: Sketch of the appearance of a hanging node $1 / 2$ depending on the nodes 0 and 1 for bilinear finite elements.

For ease of mesh refinement and coarsening hanging nodes are allowed in our implementation, as shown in Figure 4. The treatment of non-matching cells in the case of bilinear shape functions is sketched in Figure 5. 
Let $\left\langle\varphi_{i}\right\rangle, i=1 . . N$ denote the standard nodal basis for $V_{h}$ with dimension $N$. We define the vectors of nodal values $U, F \in \mathbb{R}^{N}$ with

$$
U_{i}=\left(\nabla u_{h}, \nabla \varphi_{i}\right), \quad F_{i}=\left(f, \varphi_{i}\right),
$$

and the corresponding matrix $A \in \mathbb{R}^{N \times N}$ with $A_{i j}=\left(\nabla \varphi_{j}, \nabla \varphi_{i}\right)$.

Since continuity of the solution is required, $u_{h}$ has to be linear along $\overline{01}$, i.e. assuming $u_{h}$ determined by $U_{0}$ and $U_{1}$ in nodes 0 and 1 , the value for $u_{h}$ in node $1 / 2$ has to be subjected to the constraint $U_{1 / 2}=0.5\left(U_{0}+U_{1}\right)$.

Then introducing the averaging operator $M$ the constraints for ensuring continuity of $u_{h}$ can be written in the form $U=M U$. The operator $M$ acts as on identity on regular points 0 and 1 and performs an averaging on hanging nodes $1 / 2$ giving the structure

$$
M=\left(\begin{array}{ccccc}
1 & & & & \\
& \ddots & & & \\
& \frac{1}{2} & 0 & \frac{1}{2} & \\
& & & \ddots & \\
& & & & 1
\end{array}\right)
$$

Consequently one has to solve $A U=F$ with the additional constraint $U=$ $M U$. Remarks on possibilities of incorporating the constraint into FE-implementations are discussed separately in a section below.

\subsection{Elements of higher order and nonconforming dis- cretisations}

The techniques described above for the case of bilinear shape functions can be extended to other situations. We discuss here the biquadratic and the nonconforming Crouzeix-Raviart element.

\subsubsection{Biquadratic}

For the biquadratic shape functions, let the vector on the edge with the hanging nodes be arranged in the form

$$
U^{T}=\left(\begin{array}{lllll}
U_{0} & U_{1 / 4} & U_{1 / 2} & U_{3 / 4} & U_{1}
\end{array}\right)
$$

(see Figure 6 , right). In order to preserve the continuity, $u_{h}$ has to be quadratic along $\overline{01}, u_{h}$ determined by $U_{0}, U_{1 / 2}$ and $U_{1}$. Hence

$$
\begin{aligned}
& U_{1 / 4}=\frac{3}{8} U_{0}+\frac{3}{4} U_{1 / 2}-\frac{1}{8} U_{1} \\
& U_{3 / 4}=-\frac{1}{8} U_{0}+\frac{3}{4} U_{1 / 2}+\frac{3}{8} U_{1}
\end{aligned}
$$


and the average operator is given by

$$
M=\left(\begin{array}{ccccc}
1 & & & & \\
3 / 8 & 0 & 3 / 4 & 0 & -1 / 8 \\
& & 1 & & \\
-1 / 8 & 0 & 3 / 4 & 0 & 3 / 8 \\
& & & & 1
\end{array}\right) \text {. }
$$

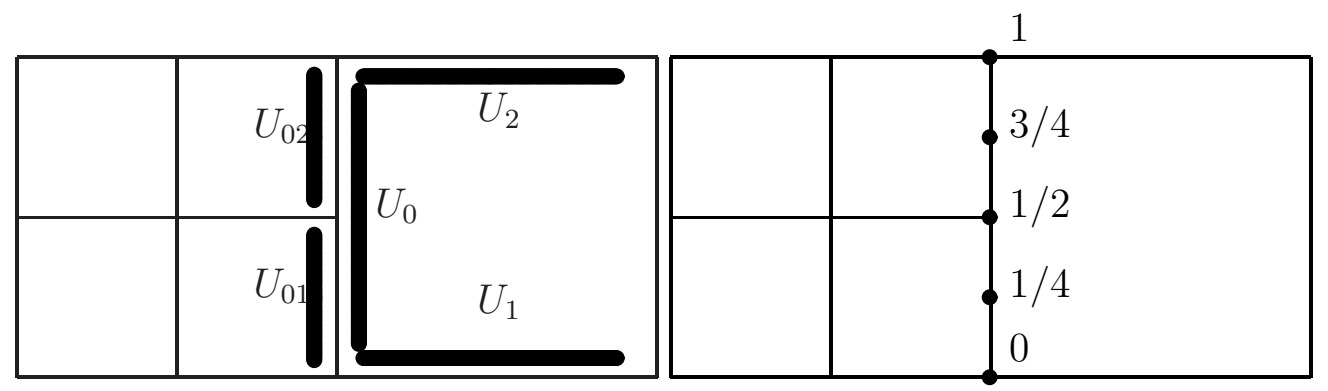

Figure 6: Hanging nodes for rotated bilinear (left) and biquadratic (right) finite elements. Bars or points indicate degrees of freedom.

\subsubsection{Rotated bilinear}

Eventually, we discuss the extension of the Crouzeix-Raviart element on triangles to quadrilaterals, i.e., the rotated bilinear element $\tilde{Q}_{1}$ analyzed by Rannacher and Turek in [6]. Analogously to the triangular elements, we take the mean value along each edge as node values. The required restrictions to the degrees of freedom in situations sketched in Figure 6 are imposed as follows. Remarking, that the node values of the rotated bilinear element are related to the element edges and are in fact the mean value along these lines, conformity with respect to the chosen discrete space is ensured by

$$
\begin{aligned}
& U_{01}=U_{0}+\frac{1}{4} U_{1}-\frac{1}{4} U_{2} \\
& U_{02}=U_{0}-\frac{1}{4} U_{1}+\frac{1}{4} U_{2} .
\end{aligned}
$$

\section{$4 \quad$ Filtering techniques}

In this section we present a possibility how the proposed treatment of hanging nodes can be incorporated into a FE-implementation. For illustration we choose a pseudo $\mathrm{C}++$ description. 


\section{Representation of an operator}

For simplicity, we assume that discretisation by the finite element method leads to a symmetric and positive definite operator $\mathrm{A}$. The $\mathrm{cg}$-method is one of the most frequently used solvers to treat such a linear system $A x=b$. In Figure 7 we sketch parts of the template implementation of the cg-method used in DEAL [2]. This solver requires only the abstract ability of the operator to do a matrix vector multiplication vmult.

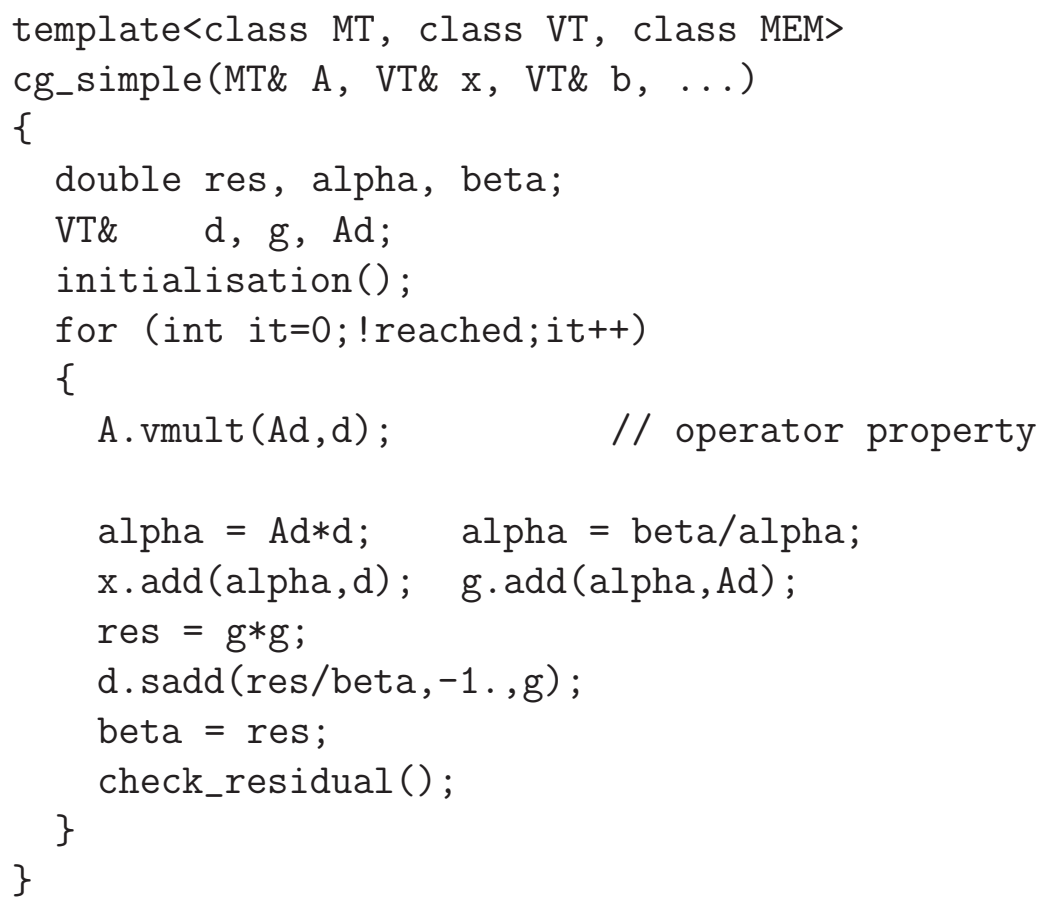

Figure 7: Pseudo code for template implementation of the cg-method

\section{Filtering techniques and hanging nodes}

In traditional approaches the treatment of Dirichlet data can be done by eliminating rows and columns of the matrix. We propose to use filtering techniques so that the representation of the operator is independent of the desired boundary values. We assume that the operator is implemented in form of a class Matrix with a default matrix-vector multiplication vmult.

The used cg-like methods are based on an update step for the solution $x$ of the form $x_{k+1}=x_{k}+\alpha_{k} d_{k}$, where $d_{k}$ is a certain correction vector. So after initialising the iteration vector with the correct Dirichlet values, we have to ensure that they are not changed during the iteration. This is simply done by modifying the matrix vector multiplication. With terms introduced above, all entries in the correction vector $d_{k}$, representing Dirichlet points, are set to 
zero after the vmult of the operator has taken place.

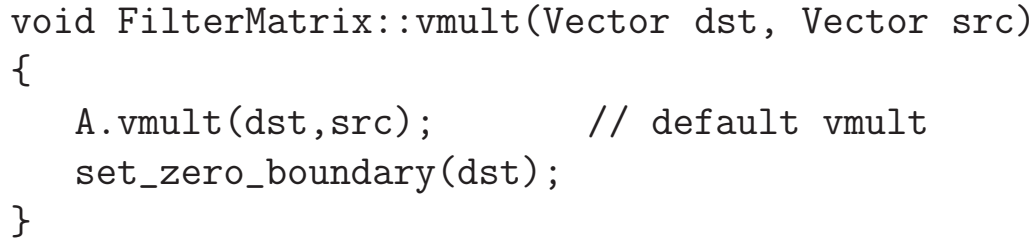

Carrying over this idea to the treatment of hanging nodes, we rewrite the discrete Poisson equation as a minimum problem

$$
\begin{gathered}
U^{T} A U-2 U^{T} F=\min , \\
U=M U
\end{gathered}
$$

or in compact form

$$
U^{T}\left(M^{T} A M\right) U-2 U^{T} M^{T} F=\min .
$$

We see that the appropriate modification of the matrix vector multiplication consists simply of the two processes $M$ and $M^{T}$ referred to as average and distribute respectively. So in our pseudo programming language the modified vmult-version looks like

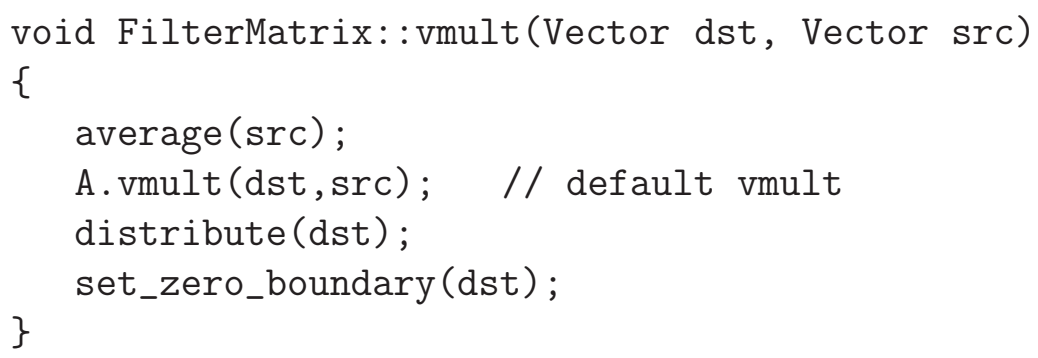

\subsection{Numerical tests}

The technique presented above has been proved to be succesful for various applications, see e.g. [1], [4], [8]. As a further example, we show some results of a comparative study of the convergence behaviour of bilinear and biquadratic shape functions on locally refined meshes. In Figure 8 (left), we show the structure of the locally refined grids produced on the basis of an a posteriori energy estimate. In Figure 8 (right) the errors measured in the energy norm on globally and locally refined grids are depicted for both discretisations. We observe the expected behaviour indicating the averaging technique to treat the hanging nodes in the correct way. 

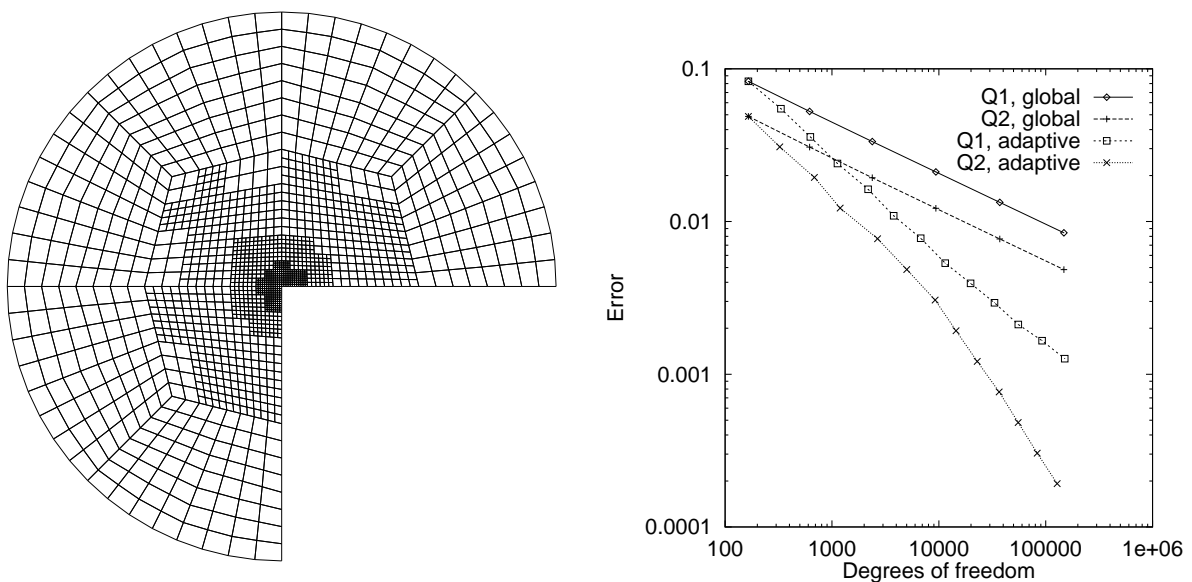

Figure 8: Adaptively refined grid for the Poisson Problem (left) and comparison of convergence behaviour of bilinear and biquadratic shape functions (right).

\section{Matrix implementation}

The main work for a finite element scheme is performed locally on the cells. Afterwards, information is transferred to the triangulation, where all degrees of freedom (DOF) are treated globally. Those processes can be described by introducing the function $I_{T}$, identifying the local numbering of $l$ DOF on a cell $T$ to $n$ DOF (numbered globally) on the triangulation.

$$
I_{T}:\{1, \cdots, l\} \rightarrow\{1, \cdots, n\} .
$$

Now, employing $I_{T}$, one can describe how local information in form of vectors and matrices becomes global. These operators are named $p_{T}$ and $P_{T}$ respectively and defined as follows.

$$
\begin{gathered}
p_{T}: \mathbb{R}^{l} \rightarrow \mathbb{R}^{n} \\
u_{n}\left(I_{T}(i)\right)=u_{l}(i), \quad i=1, \cdots, l
\end{gathered}
$$

and

$$
\begin{gathered}
P_{T}: \mathbb{R}^{l \times l} \rightarrow \mathbb{R}^{n \times n} \\
A_{n}\left(I_{T}(i), I_{T}(j)\right)=A_{l}(i, j), \quad i, j=1, \cdots, l .
\end{gathered}
$$

The traditional approach to represent the operator is the use of a classical matrix. All local information $A_{T}$ on a cell $T$ is saved in a global matrix by

$$
A=\sum_{T} P_{T} A_{T}
$$


But, within an iterative processes the result $A x$ of a matrix-vector-multiplication is required only, i.e., based on

$$
A x=\sum_{T} p_{T} A_{T} p_{T}^{-1} x
$$

$A x$ may be interpreted as the sum of results of local matrix-vector-multiplications on cells $T$.

\subsection{Evaluation}

Here, we discuss, how the concept of employing local matrices performs.

More operations: First, one observes that according to the ratio

$$
\frac{\text { entries per line in } A_{T} \times \text { cells per node }}{\text { entries per line in } \mathrm{A}}
$$

which is $16 / 9$ and $64 / 27$ for quads and hexs on tensorproduct meshes, the number of iterations per matrix-vector-multliplication increases.

Higher hardware performance: On the other hand, a higher hardware performance is achieved. This is illustrated below by evaluating MFLOP $/ \mathrm{s}$ rates for a standard 3D configuration in linear elasticity, yielding an overall speed-up.

\begin{tabular}{|r|c|c|c|c|c|}
\hline & \multicolumn{2}{|c|}{ sparse matrix } & \multicolumn{2}{c|}{ local matrix } & \\
\hline DOF & MV-time & MFLOP & MV-time & MFLOP & Speed-up \\
\hline 375 & 0.11 & 28.04 & 0.03 & 205.64 & 3.67 \\
\hline 2187 & 0.83 & 26.43 & 0.22 & 199.43 & 3.77 \\
\hline 14739 & 6.39 & 25.85 & 1.72 & 192.07 & 3.72 \\
\hline 107811 & 93.11 & 13.76 & 17.70 & 144.79 & 5.26 \\
\hline 823875 & 778.14 & 12.97 & 145.97 & 138.29 & 5.33 \\
\hline
\end{tabular}

Table 1: MFLOP/s rates: local matrices versus global sparse matrix, SUN Fire

Memory reduction: Most parts of the hierarchical structured triangulation consist of triangles or parallelograms. Consequently, the domain transformations are nearly all linear. Computing the relevant element matrices on the lowest level, the matrices required on the working level are computed simply by scaling the available knowledge from the lowest level according to the following lemma.(see [4]) 


\begin{tabular}{|r|c|c|c|c|c|}
\hline & \multicolumn{2}{|c|}{ sparse matrix } & \multicolumn{2}{c|}{ local matrix } & \\
\hline DOF & MV-time & MFLOP & MV-time & MFLOP & Speed-up \\
\hline 375 & 0.03 & 102.82 & 0.02 & 308.46 & 1.50 \\
\hline 2187 & 0.18 & 121.88 & 0.10 & 438.75 & 1.80 \\
\hline 14739 & 1.61 & 102.60 & 1.36 & 242.91 & 1.18 \\
\hline 107811 & 13.05 & 98.19 & 8.16 & 314.07 & 1.60 \\
\hline 823875 & 101.41 & 99.53 & 64.92 & 310.95 & 1.56 \\
\hline
\end{tabular}

Table 2: MFLOP/s rates: local matrices versus global sparse matrix, PC, Pentium 4

Lemma 5.1. Let $T_{0}$ be a parallelogram of dimension $d$ and the matrix $A_{0}$ the discretisation of an arbitrary differential operator of order $\omega$ on $T_{0}$.

For a cell $T$ obtained by $\nu$-fold regular refinement of $T_{0}$ into similar cells, the according matrix is obtained by

$$
A_{T}=\left(2^{\omega-d}\right)^{\nu} A_{0}
$$

Multithreading/Parallelisation: Eventually, employing cell matrices, parallelisation of a matrix-vector-multiplication becomes straight forward indicated by the pseudo-code below.

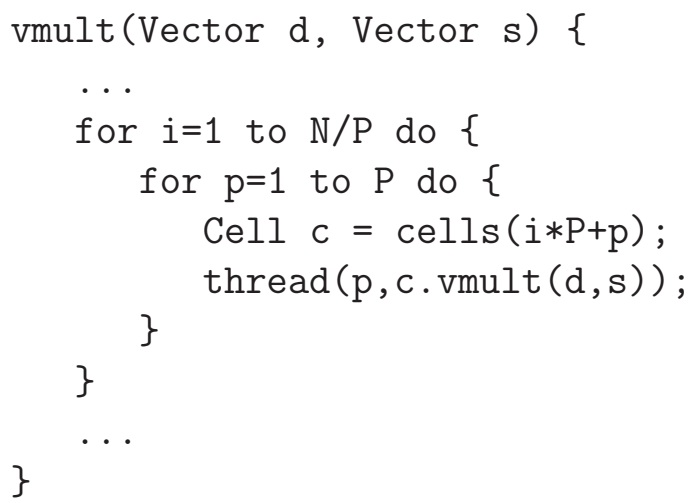

Summary: Collecting in Table 3 the considerations above, we recommend to use this concept of data locality.

\begin{tabular}{|c|l|}
\hline \multicolumn{2}{|c|}{ Cell matrices } \\
\hline- & more operations \\
\hline+ & higher hardware performance \\
\hline+ & drastical memory reduction \\
\hline+ & multithreading/parallelisation \\
\hline
\end{tabular}

Table 3: Evaluation of the performance of employing local matrices. 


\subsection{Implementation}

In order to describe the behaviour of an operator within a iterative solution process in an adequate way, the abstract ability to perform a matrix vector multiplication vmult is required only. This is realised by the class MVTriangulation in Figure 9.

\section{Classical matrix}

The traditional approach to represent the operator is the use of a classical matrix. All information is saved in a global matrix. For example one can use a SparseMatrix where only non-zero entries are stored. So our classical approach leads to the class MatrixTriangulation in Figure 9.

The required matrix vector multiplication uses the already available properties of SparseMatrix. The method A.vmult is called indirectly by the vmult of the triangulation to have the possibility to incorporate for example filter routines described above. Essentially we have the structure (see Figure 9)

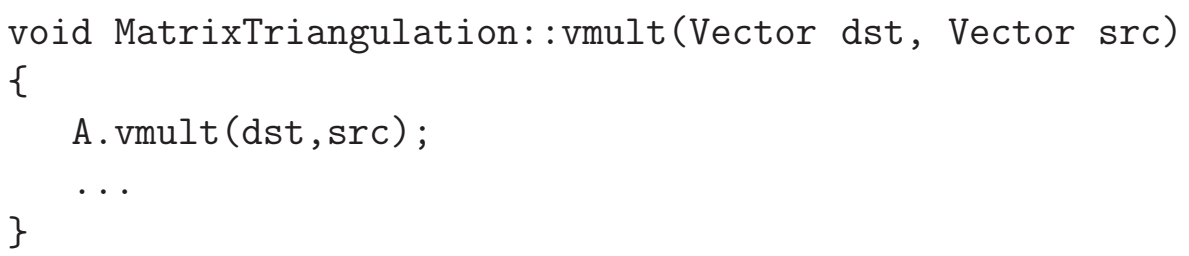

\section{Cell matrices}

As indicated above, the global operator MVTriangulation: :vmult may alternatively be implemented as a loop over all cells, where a matrix-vectormultiplication is performed locally.

This is implemented by the class LocalTriangulation in Figure 9, requiring the cells to provide an abstract vmult, which is realised by the class MVCell in Figure 10.

Next, we want to describe a concept of storing the assemble information locally. Therefore we introduce the class MatrixCell which contains the required memory in form of Matrix. Furthermore this cell is provided with a function vmult, which does the local multiplication. (see Figure 10)

Remark: During an adaptive process only parts of the whole domain are refined. Computation time is saved because the local assembling is done only on new cells and the element matrices are stored locally.

\section{Scaled matrices}

Most parts of the hierarchical structured triangulation consist of triangles or parallelograms. Consequently, the domain transformations are nearly all linear. Computing the relevant element matrices on the lowest level, the matrices required on the working level are computed simply by scaling the available knowledge from the lowest level as described above. This is implemented by the class ScaleCell in Figure 10. 
and the preconditioner. So for our purpose it is convenient to choose Jacobi-like steps as a smoother. Alternatively, fixed numbers of cg-steps may be employed to construct a local smoother on each level of the triangulation. This concept only requires the operator property too. Furthermore, we want to remark that during an adaptive process in each step we have good initial guesses for the solution, coming from computations on grids of the previous adaptive step.

\section{Elasto-plastic benchmark problem}

In order to demonstrate our adaptive FE-concepts to work within a timestepping scheme on locally varying meshes in space including hanging nodes, we show some results concerning this subject.

The fundamental problem in the flow theory of linear-elastic perfectly plastic material in classical notation reads (see, e.g., [3])

$$
\begin{aligned}
& -\operatorname{div} \sigma=f, \quad \varepsilon(\dot{u})=A \dot{\sigma}+\lambda \quad \text { in } \Omega, \\
& \lambda(\tau-\sigma) \leq 0 \quad \forall \tau \text { with } \mathcal{F}(\tau) \leq 0, \quad \lambda \dot{\sigma}=0 \quad \text { in } \Omega, \\
& \dot{u}=0 \text { on } \Gamma_{D}, \quad \sigma \cdot n=g \text { on } \Gamma_{N},
\end{aligned}
$$

where $\sigma$ and $u$ are the stress tensor and displacement vector, respectively. We assume a stress-free initial state $\sigma(0)=0$ and $u(0)=0$. This idealized model describes the deformation of an elasto-plastic body occupying a bounded domain $\Omega \subset \mathbb{R}^{d}(d=2$ or 3$)$ under the action of a body force $f$ and a surface traction $g$ along $\Gamma_{N}$. Along the remaining part of the boundary, $\Gamma_{D}=\partial \Omega \backslash \Gamma_{N}$, the body is fixed. The plastic growth is denoted by $\lambda$, and $\mathcal{F}(\cdot)$ is the (convex) von Mises yield function. We assume a quasi-static process, i.e., acceleration effects are neglected. Accordingly, the loading is prescribed in terms of functions $f(x, t)=a(t) f_{0}(x)$ and $g(x, t)=b(t) g_{0}(x)$ depending on a time-like parameter $t \in I:=[0, T]$, where the functions $a(t)$ and $b(t)$ vary only slowly (relative to the elasto-plastic changes in the model). Further, the deformation is supposed to be small, so that the strain tensor can be written as $\varepsilon(u)=\frac{1}{2}\left(\nabla u+\nabla u^{T}\right)$. The material tensor $A$ is assumed to be symmetric and positive definite. 
We apply our adaptive approach to a standard benchmark problem. A geometrically two-dimensional square disc with a hole is subjected to a constant boundary traction acting upon two opposite sides. No body force is applied, i.e., $f \equiv 0$. In the 2dimensional case we use the plane-strain approximation and assume perfectly plastic material behavior. In virtue of symmetry the consideration can be restricted to a quarter of the domain as shown in Figure 11. The height and width of the quarter corresponding to lines $\overline{45}$ and $\overline{15}$ are 100 , and the radius of the hole is 10 . The ratio of the lines $\overline{57}$ and $\overline{67}$ is $2: 1$.

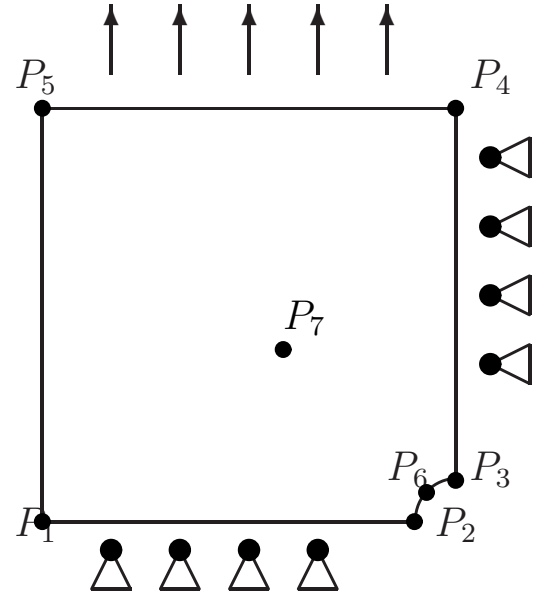

Figure 11: Geometry of the benchmark problem.

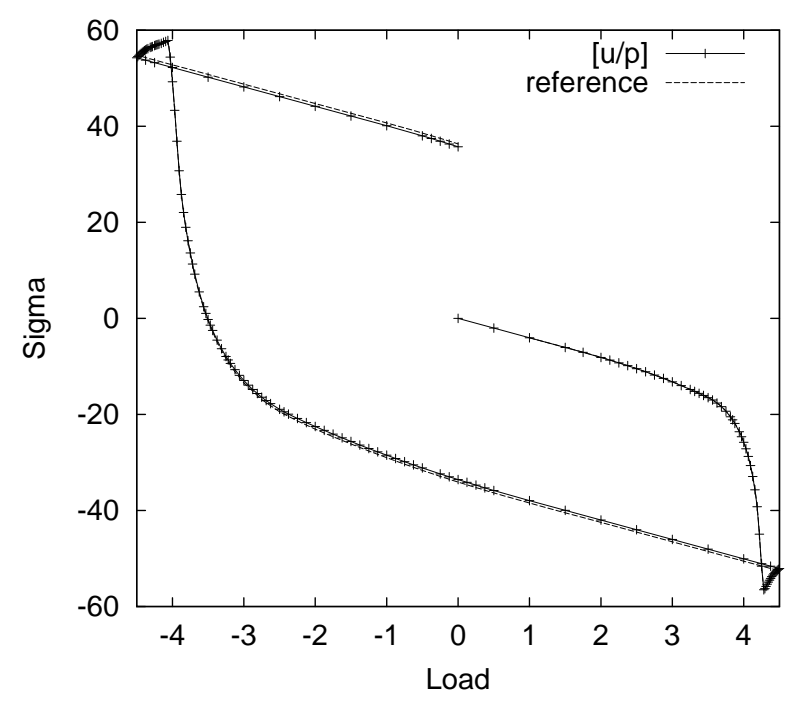

Figure 12: Plot of the reference solution (500.000 DOF) and the one obtained by our adaptive scheme (12.000 DOF).

The boundary traction is given in the form

$$
g(t)=t g_{0}, \quad g_{0}=100, \quad t \in[0,4.5] .
$$

We consider the case of a linear-elastic isotropic material law expressed in the form

$$
\sigma=C \varepsilon:=2 \mu \varepsilon^{D}(u)+\kappa \operatorname{div} u I,
$$


with material dependent constants $\mu>0$ and $\kappa>0$. Here, $C=A^{-1}$, and $\tau^{D}:=\tau-\frac{1}{3} \operatorname{tr}(\tau) I$ is the deviatoric part of a tensor $\tau$. The von Mises flow function has the form

$$
\mathcal{F}(\sigma)=\left|\sigma^{D}\right|^{2}-\sigma_{0}^{2} \leq 0
$$

In our test calculations, we have chosen the material parameters as commonly used for aluminium: $\kappa=164,206 \mathrm{~N} / \mathrm{mm}^{2}, \quad \mu=80,193.80 \mathrm{~N} / \mathrm{mm}^{2}, \sigma_{0}=$ $\sqrt{2 / 3} 450$.
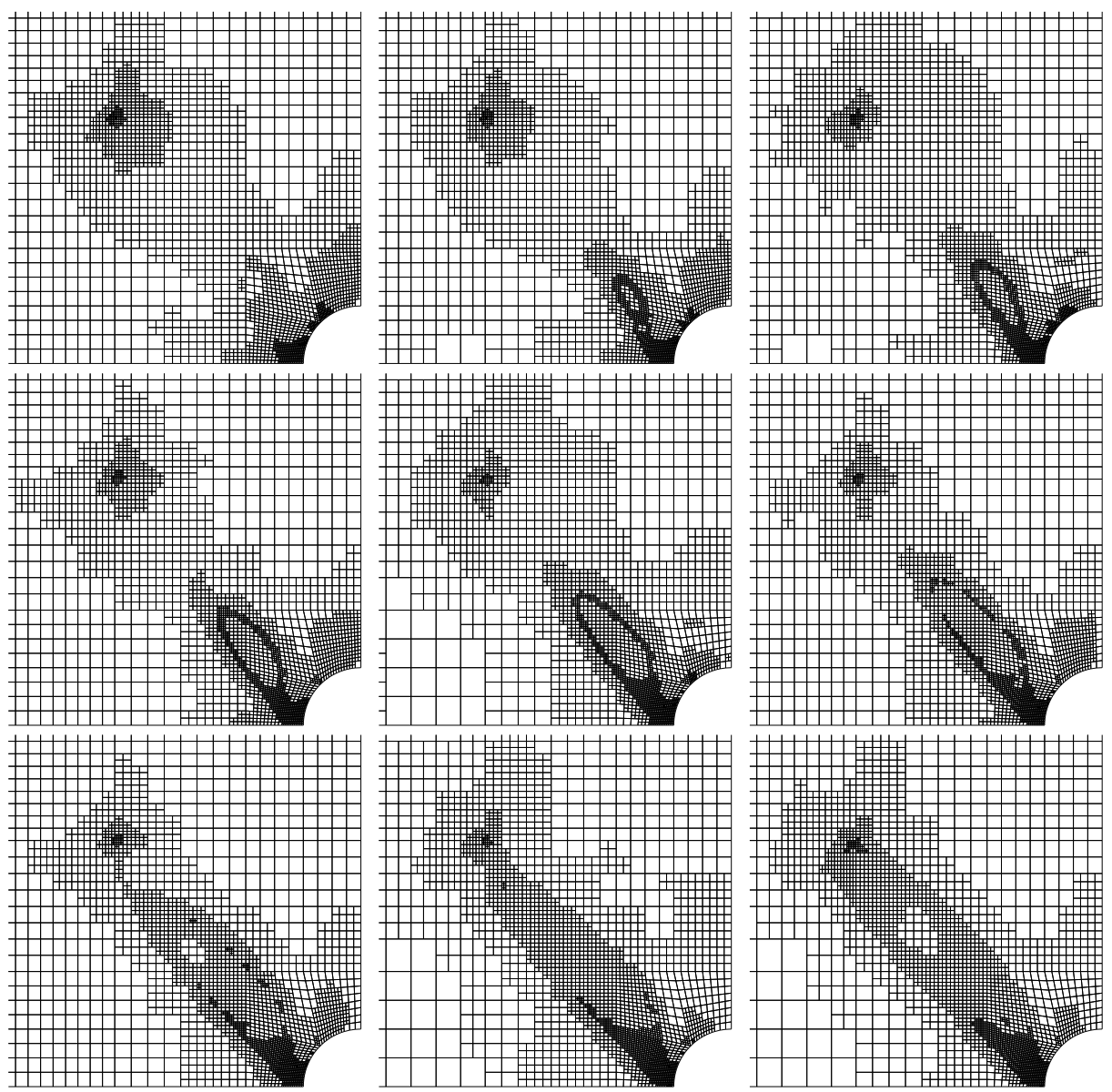

Figure 13: Sequence of the locally refined meshes including hanging nodes. Benchmark problem with zoom to one quarter of the configuration.

We evaluate values of the stresses in point $P_{7}$ under cycling loading. We compare our discretisation, which uses an a posteriori error estimate controlling the errors in time and space (c.f. [5]) to a reference solution, produced on the basis of a primal formulation approximating the displacements by the continuous, standard (bi)-linear shape functions. The pressure is treated by 
an inner variable (see [9]). About 500.000 degrees of freedom (DOF) were employed to guarentee an error below one percent. In Figure 12, there is shown the plot of the reference solution and the one obtained by our adaptive scheme using only about 12.000 DOF to reach the prescribed accuracy.

Further, Figure 13 shows a sequence of zooms into adaptively refined meshes (including hanging nodes) for computing $\sigma_{11}\left(P_{7}\right)$ over the loading path $3.6 \leq$ $t \leq 4.2$.

\section{References}

[1] R. Becker. An Adaptive Finite Element Method for the Incompressible Navier-Stokes Equations on Time-Dependent Domains. Dissertation, Institut für Angewandte Mathematik, Universität Heidelberg, 1995.

[2] DEAL. differential equations analysis library. available via http://www.math.uni-siegen.de/suttmeier/deal/deal.html, 1995.

[3] G. Duvaut and J. L. Lions. Inequalities in Mechanics and Physics. Springer, Berlin-Heidelberg-New York, 1976.

[4] G. Kanschat. Parallel and Adaptive Galerkin Methods for Radiative Transfer Problems. Dissertation, Institute for Applied Mathematics, University of Heidelberg, 1996.

[5] R. Rannacher and F.T. Suttmeier. A posteriori error estimation and mesh adaptation for finite element models in elasto-plasticity. Comput. Meth. Appl. Mech. Engrg., 176:333-361, 1999.

[6] R. Rannacher and S. Turek. Simple nonconforming quadrilateral stokes element. Num. Meth. PDE, pages 97-111, 1992.

[7] W. C. Rheinboldt and C. K. Mesztenyi. On a data structure for adaptive finite element mesh refinements. ACM Trans. Math. Software, 6:166-187, 1980 .

[8] F.T. Suttmeier. Adaptive Finite Element Approximation of Problems in Elasto-Plasticity Theory. Dissertation, Institute for Applied Mathematics, University of Heidelberg, 1996.

[9] C. Wieners. Robust multigrid methods for nearly incompressible elasticity. Computing, 64(4):289-306, 2000.

Received: May 17, 2006 